\title{
Serum neurofilament light chain as outcome marker for intensive care unit patients
}

\author{
Anna Lena Fisse ${ }^{1}\left[\right.$ - Kalliopi Pitarokoili ${ }^{1} \cdot$ David Leppert $^{2} \cdot$ Jeremias Motte $^{1} \cdot$ Xiomara Pedreiturria $^{1}$. \\ Ludwig Kappos ${ }^{2} \cdot$ Ralf Gold $^{1} \cdot$ Jens Kuhle ${ }^{2} \cdot$ Min-Suk Yoon $^{3}$
}

Received: 30 August 2020 / Revised: 13 October 2020 / Accepted: 14 October 2020 / Published online: 23 October 2020

(c) The Author(s) 2020

\begin{abstract}
Objective Neurofilament light chain (NfL) in serum indicates neuro-axonal damage in diseases of the central and peripheral nervous system. Reliable markers to enable early estimation of clinical outcome of intensive care unit (ICU) patients are lacking. The aim of this study was to investigate, whether serum NfL levels are a possible biomarker for prediction of outcome of ICU patients.

Methods Thirty five patients were prospectively examined from admission to ICU until discharge from the hospital or death. NfL levels were measured longitudinally by a Simoa assay.

Results NfL was elevated in all ICU patients and reached its maximum at day 35 of ICU treatment. Outcome determined by modified Rankin Scale at the end of the follow-up period correlated with NfL level at admission, especially in the group of patients with impairment of the central nervous system $(n=25, r=0.56, p=0.02)$.

Conclusion NfL could be used as a prognostic marker for outcome of ICU patients, especially in patients with impairment of the central nervous system.
\end{abstract}

Keywords Neurofilament $\cdot$ Intensive care unit $\cdot$ Outcome $\cdot$ Critical illness polyneuromyopathy

\section{Introduction}

Neurofilaments (NfL) are structural scaffolding proteins in neurons and are known as a biomarker reflecting neuroaxonal damage in various neurological disorders [1]. NfL is composed of subunits from Nf-L [neurofilament light], Nf-M [neurofilament middle], Nf-H [neurofilament heavy], a-internexin and peripherin [2]. Through cross-bridging and interconnecting with other components of the cytoskeleton

Anna Lena Fisse, Kalliopi Pitarokoili, Jens Kuhle, and Min-Suk Yoon contributed equally to this work.

Anna Lena Fisse

Anna.Fisse@rub.de

1 Department of Neurology, St. Josef-Hospital, RuhrUniversity Bochum, Gudrunstrasse 56, 44791 Bochum, Germany

2 Departments of Medicine and Clinical Research, Neurologic Clinic and Policlinic, University Hospital and University of Basel, Basel, Switzerland

3 Department of Neurology, Evangelisches Krankenhaus Hattingen, Hattingen, Germany (i.e. microtubules and actin filaments), they establish a regionally specialized network that is crucial for proper nerve function [2].

Elevated levels of NfL are detectable in cerebrospinal fluid and serum and were described in diseases of the central nervous system like multiple sclerosis, dementia, stroke, traumatic brain injury etc.[1, 3, 4], but also in disorders of the peripheral nervous system like Guillain-Barré syndrome and chronic inflammatory demyelination neuropathy [5-7]. Blood levels of neurofilaments were shown to monitor and predict progression in these diseases. However, NfL levels are general indicators of neuro-axonal damage irrespective of its cause.

In patients undergoing intensive care unit (ICU) treatment, biomarkers to predict clinical outcome play an important role. Reliable prediction of outcome helps to avoid both inappropriate withdrawal of life sustaining treatment in patients with good prognosis, as well as prolonged treatment in patients without chance of adequate survival. Moreover, it helps to provide correct information for patients' relatives, and, in case of a scarcity of resources, to allocate resources appropriately. A recent study showed that in case of 
resuscitation from out-of-hospital cardiac arrest more than $80 \%$ of patients admitted to an ICU have hypoxic-ischemic brain injury and about two-thirds of them die from withdrawal of life-sustaining treatment due to a predicted poor neurological outcome [8].

The aim of this study was to investigate, whether serum NfL levels are a possible biomarker for prediction of outcome of ICU patients.

\section{Methods}

\section{Subjects and patients}

This study was part of the prospective study on new approaches to critical illness polyneuromyopathy (Fisse et al., under review), approved by the ethics committee of the medical faculty of the Ruhr University Bochum (vote no. 16-5994). Written informed consent was obtained by patients or their legal representatives.

In this study, 35 patients of a neurologic internal medicine ICU in a university hospital of the Ruhr-University Bochum were prospectively examined clinically every 7 days from admission to ICU until discharge from the hospital or death. Inclusion criteria were ICU treatment with mechanical ventilation or catecholamine therapy for at least $24 \mathrm{~h}$ or sepsis or organ failure like acute respiratory distress syndrome or dialysis. Serum samples for NfL analysis were collected after admission and every 7 days during ICU treatment.

Clinical outcome was measured using modified Rankin Scale (mRS) reflecting the degree of disability ranging from 0 (no disability) to 6 (dead) [9].

\section{NfL measurements}

Peripheral blood sampling and isolation of serum were performed according to a standardized protocol. Samples were stored at $-80{ }^{\circ} \mathrm{C}$. NfL levels were measured at the Laboratory of University Hospital Basel by a Simoa assay. The complete protocol is described elsewhere [10]. Samples were coded randomly and were analyzed blinded for patient's group and outcome.

\section{Detection of patients with critical illness polyneuromyopathy}

Patients received nerve conduction studies and electromyography to detect a possible influence of critical illness polyneuromyopathy (CIPNM) on NfL levels.

CIPNM was defined as,

- Deterioration of the compound motor action potential (CMAP) amplitude during ICU stay by more than $50 \%$ and more than $1 \mathrm{mV}$ compared to the baseline nerve conduction study (NCS) in at least one leg nerve, or,

- Presence of pathological spontaneous activity in the electromyography (EMG).

To show longitudinal changes in severity of NCS and EMG pathologies, the CIPNM severity score was calculated (Fisse et al., under review):

Regarding NCS of the fibular nerve:

- 1 point for demyelinating characteristics (reduced CV, prolonged DML, prolonged F-wave latency, and conduction block);

- 1 point for distal CMAP amplitude reduction below the lower limit of normal;

- 1 additional point for distal CMAP amplitude reduction $>20 \%$;

- 1 additional point for distal CMAP amplitude reduction $>50 \%$;

- 1 additional point for distal CMAP amplitude reduction $>70 \%$;

- 1 additional point for lack of distal CMAP.

Regarding EMG of the tibialis anterior muscle:

- 1 point for pathological spontaneous activity in $\leq 5$ of 10 tested needle layers;

- 2 points for pathological spontaneous activity in $>5$ of 10 tested needle layers.

\section{Statistics}

Statistical analyses were performed using Graph Pad Prism 8 (GraphPad Software Inc., San Diego, California, USA) and IBM SPSS Statistics 25.0.0.0 (IBM Corporation, Armonk, New York, USA). Absolute data are presented as mean \pm SD or as median with range, lower and upper quartile. Differences between groups were tested by Mann-Whitney $U$ test, $t$ test or Chi-squared test as applicable. Probability levels ( $p$ values) are indicated as *, if $p<0.05$.

\section{Results}

\section{Clinical data}

In 6 of the 35 patients only baseline, but not follow-up examination was possible due to death or discharge to another hospital within the first few days after admission. Mean age was $65 \pm 12$ years. Sepsis in $45 \%$, resuscitation in $45 \%$, and stroke or intracranial hemorrhage in $28 \%$ of patients were most frequent diagnoses. Central nervous system involvement (e.g. brain death after resuscitation, encephalopathy 
in sepsis, acute cerebral infarction) occurred in 22 (76\%) of patients. Median follow-up time was 26 days (range 5-126, interquartile range 11-57). Further baseline and disease characteristics like sedation and vasopressor use are shown in Table 1.

Median mRS at the end of the follow-up period up as outcome marker of all patients was 5 (range 1-6, interquartile range 2). Median duration of ICU stay was 22 days (range 8-69, interquartile range: 21 ). Table 2 gives further details on outcome.

\section{$\mathrm{NfL}$ as outcome marker for ICU patients}

Outcome determined by mRS at the end of the follow -period (median 26 days) correlated with $\mathrm{NfL}$ level at admission in all ICU patients $(r=0.58, p=0.05$, Spearman correlation, Fig. 1). Median NfL in all ICU patients after admission was $231 \mathrm{pg} / \mathrm{ml}$. During the ICU stay, $\mathrm{NfL}$ reached its maximum at day 35 with a median $\mathrm{NfL}$ of $12,116 \mathrm{pg} / \mathrm{ml}$
Table 2 Outcome characteristics of study population (Fisse el al., under review)

\begin{tabular}{ll}
\hline & $\begin{array}{l}\text { All } \\
\text { patients } \\
(n=29)\end{array}$ \\
\hline Death, $n(\%)$ & $11(38)$ \\
Outcome mRS, median (IQR) & $5(2)$ \\
Outcome GCS, median (IQR) & $7(10)$ \\
Duration of stay at ICU in days, median (IQR) & $22(21)$ \\
Duration of stay in hospital in days, median (IQR) & $30(30)$ \\
Duration of ventilation in days, median (IQR) & $11(18)$ \\
Duration from mechanical ventilation to assisted sponta- & $6(3)$ \\
$\quad$ neous ventilation in days, median (IQR) & $12(41)$ \\
\hline Tracheostoma, $n(\%)$ &
\end{tabular}

$m R S$ modified Rankin Scale, GCS Glasgow coma scale, ICU intensive care unit
Table 1 Baseline characteristics of study population (Fisse el al., under review)

\begin{tabular}{|c|c|}
\hline & All patients $(n=29)$ \\
\hline \multicolumn{2}{|l|}{ Demographics } \\
\hline Age in years, mean $(\mathrm{SD})$ & $65(12)$ \\
\hline Women, $n(\%)$ & $10(35)$ \\
\hline Body mass index, mean (SD) & $25(5)$ \\
\hline \multicolumn{2}{|l|}{ Disease characteristics } \\
\hline Disease duration until inclusion in days, median (IQR) & $6(8)$ \\
\hline Days of sedation, median (IQR) & $12(15)$ \\
\hline Days of vasopressor-use, median (IQR) & $8(13)$ \\
\hline mRS at inclusion, median (IQR) & $5(0)$ \\
\hline GCS at inclusion, median (IQR) & $3(2)$ \\
\hline SOFA at inclusion, median (IQR) & $9(4)$ \\
\hline Maximum SOFA during ICU stay, median (IQR) & $10(3)$ \\
\hline Sepsis, $n(\%)$ & $13(45)$ \\
\hline Reanimation, $n(\%)$ & $13(45)$ \\
\hline Stroke or intracranial hemorrhage, $n(\%)$ & $8(28)$ \\
\hline Any primary CNS disease, $n(\%)$ & $19(66)$ \\
\hline Follow up time in days, median (IQR) & $26(46)$ \\
\hline \multicolumn{2}{|l|}{ Laboratory values at inclusion, mean (SD) } \\
\hline Leukocytes in $1000 / \mu l$ & $10.9(4.7)$ \\
\hline Thrombocytes in $1000 / \mu 1$ & $250(142)$ \\
\hline Haemoglobin in $\mathrm{g} / \mathrm{dl}$ & $10.4(2.6)$ \\
\hline Creatine kinase in $U / 1$ & 1048 (2359) \\
\hline Procalcitonin in $\mathrm{ng} / \mathrm{ml}$ & $1.9(5.0)$ \\
\hline Glucose in mg/dl & $156(75)$ \\
\hline C-reaktive protein in $\mathrm{mg} / \mathrm{l}$ & $122(101)$ \\
\hline Creatinine in mg/dl & $1.1(0.8)$ \\
\hline Bilirubin in mg/dl & $0.7(0.8)$ \\
\hline Oxygen partial pressure $(\mathrm{paO} 2)$ in $\mathrm{mmHg}$ & $109(35)$ \\
\hline
\end{tabular}

Mrs modified Rankin Scale, GCS Glasgow coma scale, SOFA sepsis-related organ failure assessment score, $I C U$ intensive care unit, $C N S$ central nervous system 


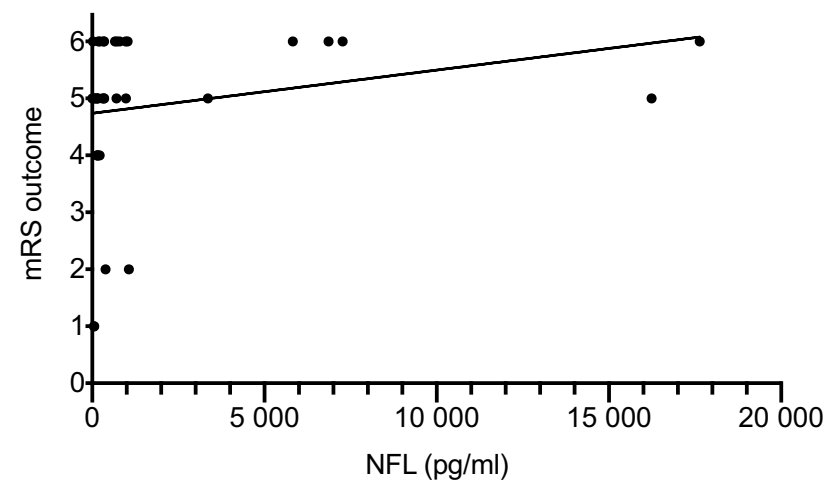

Fig. 1 Correlation between NfL at admission and outcome mRS at end of follow-up in the whole study group $(r=0.58, p=0.05)$

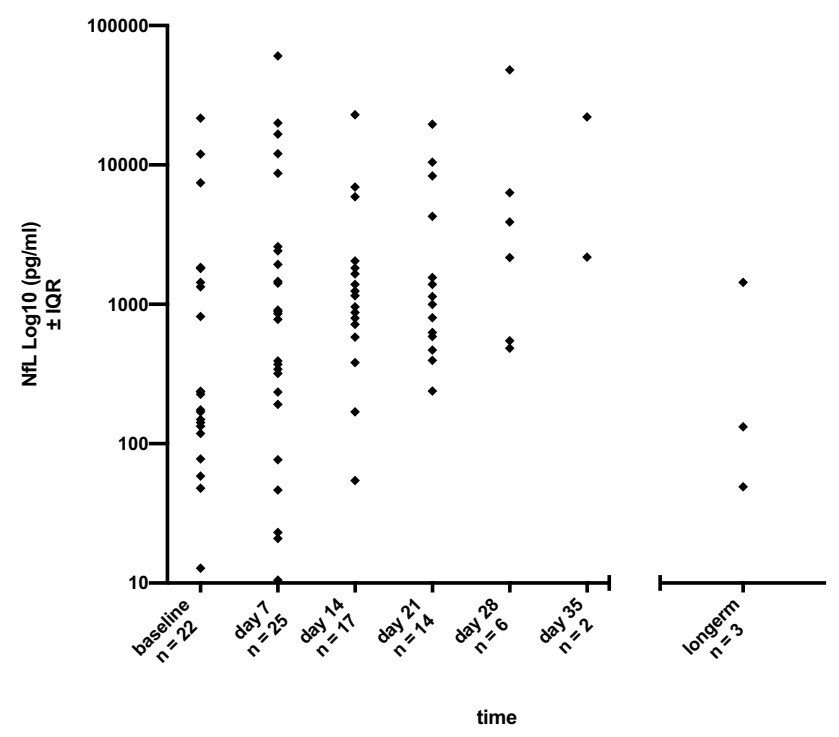

Fig. 2 NfL course during ICU stay shown as $\log 10$ of median. Highest NfL levels were found on day 35

(Fig. 2; Table 3). Over time, outcome also correlated to NfL levels at day 7 ( $r=0.49, p=0.01)$, not on day 14 , day 21 and day 28. Serum samples at day 35 were only available from 2 patients and serum samples for long term follow-up after 6 months were only available from 3 patients.

\section{$\mathrm{NfL}$ in patients with impairment of the central nervous system (CNS)}

In the group of patients with CNS damage (e.g. brain death, encephalopathy, acute cerebral infarction, $n=22$ ) NfL values at admission were significantly higher compared to patients without CNS damage $(n=7)$. Median NfL at admission in patients with CNS damage was $528 \mathrm{pg} / \mathrm{ml}$ versus $83 \mathrm{pg} / \mathrm{ml}$ in patients without CNS damage ( $p=0.02$, Fig. 3 , Table 3 ).
In this group of patients with CNS disease, there was a significant correlation between the outcome defined by $\mathrm{mRS}$ at the last follow-up and the NfL level at admission $(r=0.56$, $p=0.02$ ). Again, this correlation was also found on day 7 $(r=0.58, p=0.01)$ and not in the following weeks.

In patients without CNS impairment, correlation between $\mathrm{NfL}$ at baseline and outcome NfL was not statistically significant $(r=0.17, p=0.65)$. In these patients without CNS disease median $\mathrm{NfL}$ at admission was $83 \mathrm{pg} / \mathrm{ml}$ which above range of standard values in healthy volunteers $[10,11]$.

\section{NfL in CIPNM}

69\% ( $n=20)$ of the 29 patients developed CIPNM, while 9 patients did not develop CIPNM. No significant differences in NfL levels between patients with CIPNM and without CIPNM were found at any timepoint. Excluding patients with CNS disease, only 6 patients with CIPNM remained. In these 6 patients with CIPNM and without CNS disease median NfL at admission was $48 \mathrm{pg} / \mathrm{ml}$, which is in range of standard values in healthy volunteers $[10,11]$ and reached its maximum on day 28 with a median of $2163 \mathrm{pg} / \mathrm{ml}$.

\section{Discussion}

Our main finding, the correlation of NfL at baseline with the outcome mRS in ICU patients with CNS disease suggest that NfL could serve as a possible prognostic marker for these ICU patients. In some specific CNS diseases, correlation of $\mathrm{NfL}$ with clinical outcome was reported before, i.e. in spinal cord injury [12], traumatic brain injury [13] and multiple sclerosis [6]. The special feature of our cohort is that a broad spectrum of different neurological and non-neurological diseases was included, and NfL correlated with outcome not only in CNS-specific diseases leading to ICU treatment like for example stroke, but also in all other patients with CNS symptoms like encephalopathy and delirium irrespective of the underlying disease. If confirmed in further studies, NfL could serve as a general prognostic marker for ICU patients with CNS symptoms, like neuron-specific enolase in resuscitated patients [14]. This would be helpful in clinical context, as the extent of the neurological involvement in analgosedated patients is difficult to assess. As NfL is specific for neuronal damage, the increased $\mathrm{NfL}$ values in patients without clinically detectable CNS involvement compared to healthy subjects suggest that there is neglected neurological involvement also in these patients. Correlation of NfL levels with outcome shows that the degree of neurological involvement strongly influences outcome. This emphasizes the importance of interdisciplinary patient care in an intensive care unit to enable preventive or early 
Table 3 NfL values (pg/ml) during ICU treatment

\begin{tabular}{llllllll}
\hline & Baseline & Day 7 & Day 14 & Day 21 & Day 28 & Day 35 & Longterm \\
\hline All patients & & & & & & & \\
Available samples & 22 & 25 & 17 & 14 & 6 & 2 & 3 \\
Median & 231 & 857 & 1151 & 1067 & 3031 & 12,116 & 132 \\
Minimum & 13 & 11 & 54 & 238 & 484 & 2178 & 49 \\
Maximum & 21,689 & 60,591 & 22,909 & 19,608 & 48,101 & 22,054 & 1435 \\
Lower quartile & 130 & 213 & 650 & 558 & 531 & 2178 & 49 \\
Upper quartile & 1813 & 2506 & 1932 & 5290 & 16,759 & & \\
CNS disease & & & & & & & \\
Available samples & 18 & 19 & 11 & 10 & 4 & 2 & 2 \\
Median & 528 & 1417 & 1388 & 1263 & 5106 & 12,116 & 784 \\
Minimum & 59 & 11 & 169 & 238 & 547 & 2178 & 132 \\
Maximum & 21,689 & 60,591 & 22,909 & 19,608 & 48,101 & 22,054 & 1435 \\
Lower quartile & 147 & 342 & 797 & 758 & 1385 & 2178 & 132 \\
Upper quartile & 1827 & 8702 & 5916 & 8864 & 37,654 & & \\
No CNS disease & & & & & & & 4 \\
Available samples & 4 & 6 & 6 & 4 & 2 & 0 & 1 \\
Median & 83 & 183 & 866 & 529 & 1323 & & 49 \\
Minimum & 13 & 21 & 54 & 396 & 484 & & 49 \\
Maximum & 175 & 910 & 1819 & 4275 & 2163 & & 49 \\
Lower quartile & 22 & 23 & 300 & 414 & 484 & & 49 \\
Upper quartile & 161 & 522 & 1387 & 3353 & & & 49 \\
\hline & & & & & & & \\
\hline
\end{tabular}

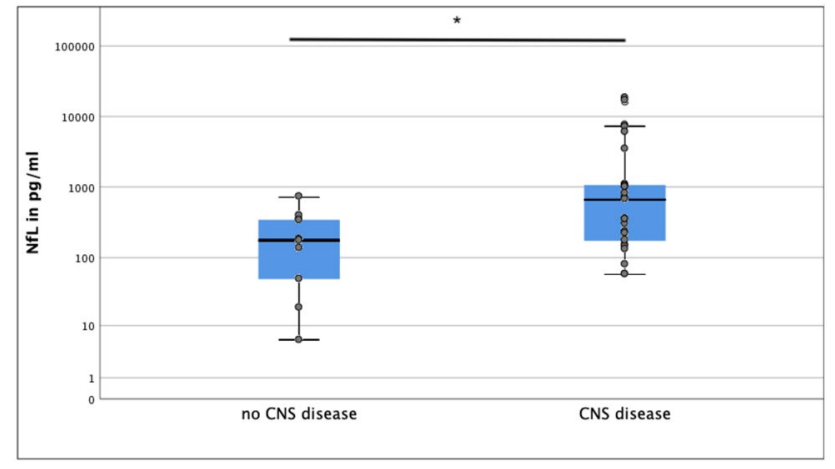

Fig. 3 NfL levels in patients with and without CNS disease

neurological therapies if required (i.e. preservation of the circadian rhythm or physiotherapy).

Long-term data of NfL over time have shown that changes in NfL levels are associated with disability worsening in multiple sclerosis. A reduction of NfL levels at 6-month intervals was reported in multiple sclerosis patients treated with high-potency therapies [15]. To our knowledge, only scarce studies showed NfL levels in acute illness during the first weeks [16]. Our results with correlation of first NfL level with outcome and increasing NfL during the time of ICU stay suggests that NfL corresponds to the degree of axonal damage at the beginning of an acute axonal lesion, and then probably accumulates over a period of a few weeks as the correlation fades over time. The peak of NfL at day 35 probably means that for these two patients the CNS damage (one with stroke, the other one with hyperprolinemia and status epilepticus) was progressive and NfL accumulated. This could be due to an accordingly long serum half-life of several weeks which was also found in other studies about NfL kinetics [4, 17].

NfL shows axonal damage but is not specific for central nervous system. Polyneuropathies leading to increased NfL levels were described before [6, 7]; however, in our study groups, we did not find elevated NfL levels in patients with CIPNM. Critically ill ICU patients often not only have CIPNM but also CNS impairment, for example encephalopathy, which leads to high NfL levels, probably as CNS damage includes much more cells then the peripheral nerves. $\mathrm{NfL}$ as marker for CIPNM should therefore be examined in patients with CIPNM without CNS impairment to investigate whether NfL could be a marker for CIPNM. In our study group, however, only 6 patients with CIPNM had no CNS impairment. In these 6 patients, $\mathrm{NfL}$ increase during ICU treatment with maximum on day 28 could imply that $\mathrm{NfL}$ could still be a possible biomarker in CIPNM.

A major limitation of this study is the small number of patients. Especially NfL measurements from day 28 onwards could only be performed in only few patients, as a lot of patients had a severe disease course and died in the first weeks of ICU treatment. Further studies are, therefore, necessary to confirm our longitudinal findings. Moreover, mRS 
was used as outcome marker, which originally was developed as an instrument for the outcome of stroke patients. However, in our view, the $\mathrm{mRS}$ represents the everyday life impairment, not only through stroke, but also through other impairments.

\section{Conclusion}

NfL could be used as a prognostic marker for outcome of ICU patients, especially in patients with CNS impairment.

Author contributions ALF: study design, data collection, drafting, and revising the manuscript. KP: study design, data collection, and revising the manuscript. DL: drafting and revising the manuscript. JM: drafting and revising the manuscript. XP: analysis of neurofilament and data collection. LK: analysis of neurofilament, drafting, and revising the manuscript. RG: critical comments during data collection and manuscript revision. M-SY: study design, critical comments during data collection and manuscript revision. JK: analysis of neurofilament, drafting and revising the manuscript.

Funding Open Access funding enabled and organized by Projekt DEAL. This study was funded by Georgius Agricola Stiftung Ruhr, Bochum, Germany.

Data availability Data and material are available on reasonable request from the corresponding author.

Code availability Not applicable.

\section{Compliance with ethical standards}

Conflicts of interest Anna Lena Fisse: received honoraria and travel grants from Novartis AG, Sanofi and Eisai GmbH, none related to this work. Owns shares of Fresenius SE \& Co., Gilead Sciences, Medtronic PLC and Novartis AG. Kalliopi Pitarokoili: received travel grants and speakers' honoraria from Novartis, Biogen idec, Teva, Bayer Schering, CSL Behring, Celgene and Grifols all not related to the manuscript. David Leppert: none related to this work. Jeremias Motte: received travel grants from Biogen idec, Novartis AG, Teva and Eisai GmbH, his research is funded by Klaus Tschira Foundation and Ruhr-University, Bochum (FoRUM-program); none related to this work. Xiomara Pedreiturria: none related to this work. Ludwig Kappos: none related to this work. Ralf Gold: received consultation fees and speaker honoraria from Bayer Schering, Biogen idec, Merck Serono, Novartis, SanofiAventis and TEVA. He also acknowledges grant support from Bayer Schering, Biogen idec, Merck Serono, Sanofi-Aventis and TEVA, none related to this manuscript. Min-Suk Yoon: received speakers' honoraria from CSL Behring, Grifols and scientific grant from CSL Behring. Jens Kuhle: received speaker fees, research support, travel support, and/or served on advisory boards by ECTRIMS, Swiss MS Society, Swiss National Research Foundation (320030_189140/1), University of Basel, Bayer, Biogen, Genzyme, Merck, Novartis, Protagen AG, Roche, Teva.

Ethical approval The ethics committee of the medical faculty of the Ruhr University Bochum approved our study protocol (vote no. 16-5994) and this study has, therefore, been performed in accordance with the ethical standards laid down in the 1964 Declaration of Helsinki and its later amendments.
Informed Consent Written informed consent was obtained by patients or their legal representatives prior to their inclusion in the study.

Consent for publication All authors read and approved the final manuscript.

Open Access This article is licensed under a Creative Commons Attribution 4.0 International License, which permits use, sharing, adaptation, distribution and reproduction in any medium or format, as long as you give appropriate credit to the original author(s) and the source, provide a link to the Creative Commons licence, and indicate if changes were made. The images or other third party material in this article are included in the article's Creative Commons licence, unless indicated otherwise in a credit line to the material. If material is not included in the article's Creative Commons licence and your intended use is not permitted by statutory regulation or exceeds the permitted use, you will need to obtain permission directly from the copyright holder. To view a copy of this licence, visit http://creativecommons.org/licenses/by/4.0/.

\section{References}

1. Khalil M et al (2018) Neurofilaments as biomarkers in neurological disorders. Nat Publ Group 14:577-589

2. Yuan A, Rao MV, Veeranna Nixon RA (2017) Neurofilaments and neurofilament proteins in health and disease. Cold Spring Harb Perspect Biol 9:a018309

3. Inoue $\mathrm{R}$ et al (2017) Direct evidence of central nervous system axonal damage in patients with postoperative delirium: a preliminary study of pNF-H as a promising serum biomarker. Neurosci Lett 653:39-44

4. Thelin EP et al (2017) Serial sampling of serum protein biomarkers for monitoring human traumatic brain injury dynamics: a systematic review. Front Neurol 8:362-423

5. Petzold A et al (2006) CSF neurofilament levels: a potential prognostic marker in Guillain-Barré syndrome. Neurology 67:1071-1073

6. Mariotto S et al (2018) Serum and cerebrospinal neurofilament light chain levels in patients with acquired peripheral neuropathies. J Peripher Nerv Syst. https://doi.org/10.1111/jns.12279

7. van Lieverloo GGA et al (2019) Serum neurofilament light chain in chronic inflammatory demyelinating polyneuropathy. J Peripher Nerv Syst. https://doi.org/10.1111/jns.12319

8. Sandroni C et al (2020) Prediction of poor neurological outcome in comatose survivors of cardiac arrest: a systematic review. Intens Care Med 46:1803-1851

9. van Swieten JC, Koudstaal PJ, Visser MC, Schouten HJ, van Gijn J (1988) Interobserver agreement for the assessment of handicap in stroke patients. Stroke 19:604-607

10. Disanto G et al (2017) Serum Neurofilament light: a biomarker of neuronal damage in multiple sclerosis. Ann Neurol 81:857-870

11. Barro $\mathrm{C}$ et al (2018) Serum neurofilament as a predictor of disease worsening and brain and spinal cord atrophy in multiple sclerosis. Brain 141:2382-2391

12. Kuhle J et al (2015) Serum neurofilament light chain is a biomarker of human spinal cord injury severity and outcome. J Neurol Neurosurg Psychiatry 86:273

13. Shahim $P$ et al (2016) Serum neurofilament light protein predicts clinical outcome in traumatic brain injury. Nat Publ Group 6:36791

14. Zandbergen EGJ et al (2006) Prediction of poor outcome within the first 3 days of postanoxic coma. Neurology 66:62-68

15. Cantó E et al (2019) Association between serum neurofilament light chain levels and long-term disease course among patients 
with multiple sclerosis followed up for 12 years. Jama Neurol 76:1359-1366

16. Saller T et al (2019) A case series on the value of tau and neurofilament protein levels to predict and detect delirium in cardiac surgery patients. Biomed Pap 163:241-246
17. Bergman J et al (2016) Neurofilament light in CSF and serum is a sensitive marker for axonal white matter injury in MS. Neurol R Neuroimmunol Neuroinflammation 3:e271 